\title{
Racial and Socioeconomic Disparities in Utilization of Telehealth in Patients with Liver Disease During COVID-19
}

\author{
Kara Wegermann ${ }^{1} \cdot$ Julius M. Wilder $^{1,3} \cdot$ Alice Parish $^{2} \cdot$ Donna Niedzwiecki $^{2} \cdot$ Ziad F. Gellad $^{1,3} \cdot$ Andrew J. Muir $^{1,3}$. \\ Yuval A. Patel ${ }^{1}$
}

Received: 24 September 2020 / Accepted: 10 January 2021 / Published online: 28 January 2021

(c) The Author(s), under exclusive licence to Springer Science+Business Media, LLC part of Springer Nature 2021

\begin{abstract}
Background and Aims The coronavirus disease 2019 (COVID-19) pandemic resulted in a rapid expansion of telehealth services in hepatology. However, known racial and socioeconomic disparities in internet access potentially translate into barriers for the use of telehealth, particularly video technology. The specific aim of this study was to determine if disparities in race or socioeconomic status exist among patients utilizing telehealth visits during COVID-19.

Methods We performed a retrospective cohort study of all adult patients evaluated in hepatology clinics at Duke University Health System. Visit attempts from a pre-COVID baseline period (January 1, 2020 through February 29, 2020; $n=3328$ ) were compared to COVID period (April 1, 2020 through May 30, 2020; $n=3771$ ).

Results On multinomial regression modeling, increasing age was associated with higher odds of a phone or incomplete visit (canceled, no-show, or rescheduled after May 30,2020), and non-Hispanic Black race was associated with nearly twice the odds of completing a phone visit instead of video visit, compared to non-Hispanic White patients. Compared to private insurance, Medicaid and Medicare were associated with increased odds of completing a telephone visit, and Medicaid was associated with increased odds of incomplete visits. Being single or previously married (separated, divorced, widowed) was associated with increased odds of completing a phone compared to video visit compared to being married.

Conclusions Though liver telehealth has expanded during the COVID-19 pandemic, disparities in overall use and suboptimal use (phone versus video) remain for vulnerable populations including those that are older, non-Hispanic Black, or have Medicare/Medicaid health insurance.
\end{abstract}

Keywords Telehealth $\cdot$ Telemedicine $\cdot$ Health disparity $\cdot$ Medicaid $\cdot$ Marital status

Supplementary Information The online version contains Abbreviations

COVID-19 Coronavirus disease 2019

SARS-CoV-2 Severe acute respiratory syndrome coronavirus 2

supplementary material available at https://doi.org/10.1007/s1062

0-021-06842-5.

Yuval A. Patel

yuval.patel@duke.edu

Kara Wegermann

kara.wegermann@duke.edu

Julius M. Wilder

julius.wilder@duke.edu

Alice Parish

alice.parish@duke.edu

Donna Niedzwiecki

donna.niedzwiecki@duke.edu

Ziad F. Gellad

ziad.gellad@duke.edu
Andrew J. Muir

andrew.muir@duke.edu

1 Division of Gastroenterology, Department of Medicine, Duke University School of Medicine, Box 3913, Durham, NC 27710, USA

2 Department of Biostatistics and Bioinformatics, Duke University, Durham, USA

3 Duke Clinical Research Institute, Durham, USA 


\section{CMMS Centers for Medicare and Medicaid Services \\ APP \\ Advanced practice provider}

\section{Introduction}

Coronavirus disease 2019 (COVID-19), a potentially fatal infection caused by severe acute respiratory syndrome coronavirus 2 (SARS-CoV-2), changed healthcare practices in the US and around the globe. In particular, the COVID19 pandemic produced a large increase in use of telehealth as a safer alternative to care that reduces exposure of both patients and healthcare providers to the virus [1]. The US Department of Health and Human Services defines telehealth as "The use of electronic information and telecommunications technologies to support and promote long-distance clinical health care, patient and professional health-related education, public health and health administration" [2]. This has transformed the field of hepatology significantly, in which in-person visits are a hallmark of chronic disease management.

Telehealth has been discussed and promoted as a way to increase access to hepatology care among specific populations (e.g., those living in rural areas who must travel hours to tertiary care centers) and to improve chronic disease management [3]. Previously, liver telehealth was reserved for specific clinical situations, such as hepatitis $\mathrm{C}$ treatment, hepatocellular carcinoma, or post-liver transplant monitoring [4]. A major limitation to expansion of telehealth in hepatology has been reimbursement. This barrier was lifted with the Centers for Medicare \& Medicaid Services (CMMS) decisions to waive requirements such as residence in a rural area, as well as the decision to increase reimbursement, such that telephone visits are now reimbursed equally to video and in-person visits [5]. Recently published data indicate cost-effectiveness for telemedicine tools in the management of inflammatory bowel disease, but more data are needed in hepatology [6]. As such, telehealth has become a vital tool in delivering care to patients with liver disease since the emergence of COVID-19. Combined with remote monitoring systems, many centers have shifted care toward non-face-to-face methods [7].

There is evidence that video technology has advantages compared to telephone for telemedicine clinics. Small studies indicate superiority of video technology over telephone in specific situations, for example, acute stroke, presumably because physical examination is essential [8]. A systematic review of studies published from 2000 to 2018 found that video visits were associated with fewer medication errors and higher accuracy of initial diagnosis compared to telephone visits; however, patient outcomes, including mortality, were similar [9]. In a recent study of patient satisfaction surveys in gastroenterology clinics during COVID-19, video visits were associated with a higher percentage of patients reporting that the telehealth visit was as good as, or better than, a face-to-face visit [10]. However, racial and socioeconomic disparities in internet access are well documented and may impact patients' abilities to access video visits [11]. The specific aim of this study was to determine if disparities in race or socioeconomic status exist among patients utilizing video or telephone telehealth visits during the COVID-19 era.

\section{Methods}

\section{Patients}

We performed a retrospective cohort study of all visit attempts (completed, rescheduled, canceled, or no-show) in adult hepatology clinics (general and transplant) at Duke University Health System from January 1, 2020, through May 30, 2020. We divided visits into three periods: preCOVID (January 1, 2020, through February 29, 2020), COVID (April 1, 2020, through May 31, 2020), and outside study timeframe (any date after May 31, 2020, as visits were often rescheduled months in the future). The month of March 2020 was not included in the above groupings as it reflected a transition period with the onset of COVID-19 in our local area. Visits for procedures including paracentesis or transient elastography were excluded. All patients were offered video visits first, and if the patient was unable to complete a video visit or declined, a telephone visit was offered. Video visits required internet access and a computer, tablet, or smartphone with a camera. Video visits were conducted through (1) the extended care platform using Duke Maestro Care ${ }^{\odot}$, which is based upon the EPIC $^{\odot}$ electronic health record systems platform, and required an active online patient account and internet access, or (2) the Doximity ${ }^{\odot}$ dialer video interface, which can utilize cellular data. The extended care platform was preferred, but providers were advised to switch to Doximity during the visit if technical difficulties arose that could not be resolved promptly. Inperson visits were offered only at the provider's discretion. A modified schedule was created in which providers had limited opportunity to see patients in person. The study was approved by the Duke Institutional Review Board.

\section{Visit Data}

Data on visits and patient demographics were obtained through Duke Performance Services. Demographics included patient age, sex, self-reported race and ethnicity, marital status, ZIP code of primary residence, and insurance information. We divided race/ethnicity into five categories: 
Black (Black race, non-Hispanic ethnicity), White (White race, non-Hispanic ethnicity), Hispanic (Hispanic ethnicity, any race), Other (anything other than the above, including multiracial), and Not Recorded. Education level was not included as it was significantly underreported. Insurance was classified as Medicaid, Medicare, Private insurance, Other (insurance not fitting the above categories), or Not Recorded (self-pay, uninsured, or insurance data were not available at the time of data extraction). ZIP code was converted to patient service area and was divided into four categories: within 25 miles of Duke University Hospital, within 50 miles, other North Carolina (within the state of North Carolina), and out of state (outside North Carolina). Visit-level data included type of visit (in-person, telephone or video), provider (MD or Advanced Practice Provider [APP, PA, or NP]), clinic location, and visit status (completed, canceled, no-show, or rescheduled). Patient experience survey data were also obtained for patients who completed telehealth (telephone or video) visits and included answers to a series of questions about their satisfaction with the visit.

\section{Statistical Analysis}

All analyses were done using SAS $^{\odot}$ version 9.4 (Cary, NC). Continuous variables were summarized with mean/standard deviation/median/Q1-Q3/range and categorical variables with frequency counts and percentages. The number of missing data for each variable is reported. The appropriate statistical test (Wilcoxon rank-sum or Chi-square test) was used to test for differences in characteristics. Additionally, we utilized a multinomial logistic regression model to assess characteristics associated with completion of telehealth visits during the COVID period. The three outcomes for this model were completed telephone visit, completed video visit, and incomplete visit (i.e., canceled/no-show/rescheduled). The predictors were chosen a priori: age, sex, race/ ethnicity, insurance, marital status, and patient market area (geographic indicator).

\section{Results}

A total of 13,628 visit attempts among 18 unique providers (12 physicians, 6 physician assistants) were analyzed for the study. Of these, 3238 took place during the pre-COVID period, 3771 during the COVID period, and 6619 were outside study timeframe. A comparison of visit characteristics for the pre-COVID and COVID periods is shown in Fig. 1. Overall, a similar number of visit attempts took place during these two periods (3238 for pre-COVID versus 3771 for COVID). More visits were rescheduled or canceled/no-show during the COVID period. There was a statistically significant difference in composition of patients that completed pre-COVID era visits versus COVID-era telehealth visits by sex and insurance status (Supplemental Table 1).

Table 1 compares visit attempt types during the COVID period. There was no difference in visit categories by sex. There was a statistically significant difference by age, with the median age of phone appointments being 63 years old, while the median age of video appointments was 58 years old $(p<0.001)$. There was also a significant difference by race/ethnicity $(p<0.001)$. Visit attempts for Hispanic and Other patients had the highest rates $(42 \%$ and $41 \%$, respectively) of unseen appointments during the COVID period (canceled, no-show, or rescheduled to outside study period). Black patients had the highest rate of completed telephone appointments (24\%) and lowest rate of completed video visits $(26 \%)$.
Fig. 1 Comparison of visit status between pre-COVID and COVID periods

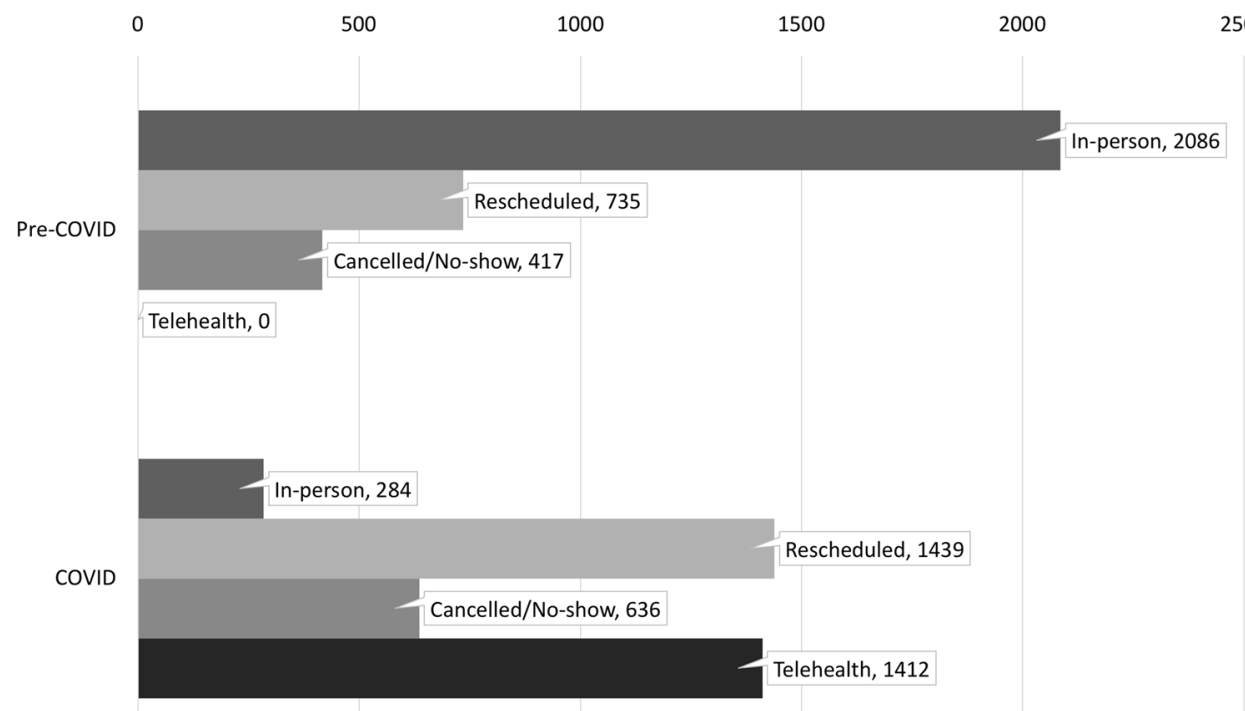


Table 1 COVID period visits by outcome

\begin{tabular}{|c|c|c|c|c|c|c|c|}
\hline Category & Variable & $\begin{array}{l}\text { Completed } \\
\text { telephone visits } \\
(N=454)\end{array}$ & $\begin{array}{l}\text { Completed } \\
\text { video visits } \\
(N=958)\end{array}$ & $\begin{array}{l}\text { Rescheduled from } \\
\text { in-person to } \\
\text { telehealth } \\
(N=473)\end{array}$ & $\begin{array}{l}\text { Canceled/no- } \\
\text { show/rescheduled } \\
\text { outside study } \\
\text { period }(N=1114)\end{array}$ & Total $(N=2999)$ & $P$ value \\
\hline Age & Median (Q1, Q3) & $63.0(56.0,70.0)$ & $58.0(46.0,67.0)$ & $60.0(49.0,68.0)$ & $61.0(51.0,69.0)$ & $60.0(50.0,68.0)$ & $<0.001^{\mathrm{a}}$ \\
\hline \multirow[t]{2}{*}{ Sex } & Female & $260(15.9 \%)$ & $520(31.8 \%)$ & $260(15.9 \%)$ & $593(36.3 \%)$ & $1633(54.5 \%)$ & \multirow[t]{2}{*}{$0.535^{\mathrm{b}}$} \\
\hline & Male & $194(14.2 \%)$ & $438(32.1 \%)$ & $213(15.6 \%)$ & $521(38.1 \%)$ & $1366(45.5 \%)$ & \\
\hline \multirow[t]{5}{*}{ Race/ethnicity } & Hispanic & $13(13.5 \%)$ & $31(32.3 \%)$ & $12(12.5 \%)$ & $40(41.7 \%)$ & $96(3.2 \%)$ & \multirow[t]{5}{*}{$<0.001^{\mathrm{b}}$} \\
\hline & Black & $131(23.6 \%)$ & $142(25.6 \%)$ & $93(16.8 \%)$ & $188(33.9 \%)$ & $554(18.5 \%)$ & \\
\hline & White & $268(13.3 \%)$ & $685(34.0 \%)$ & $324(16.1 \%)$ & $738(36.6 \%)$ & $2015(67.2 \%)$ & \\
\hline & Other & $24(13.3 \%)$ & $57(31.7 \%)$ & $25(13.9 \%)$ & $74(41.1 \%)$ & $180(6.0 \%)$ & \\
\hline & Not Recorded & $18(11.7 \%)$ & $43(27.9 \%)$ & $19(12.3 \%)$ & $74(48.1 \%)$ & $154(5.1 \%)$ & \\
\hline \multirow[t]{5}{*}{ Insurance } & Medicaid & $53(26.2 \%)$ & $50(24.8 \%)$ & $34(16.8 \%)$ & $65(32.2 \%)$ & $202(6.7 \%)$ & \multirow[t]{5}{*}{$<0.001^{\mathrm{b}}$} \\
\hline & Medicare & $266(19.5 \%)$ & $371(27.2 \%)$ & $208(15.3 \%)$ & $517(38.0 \%)$ & $1362(45.4 \%)$ & \\
\hline & Private & $94(8.0 \%)$ & $480(40.8 \%)$ & $190(16.1 \%)$ & $413(35.1 \%)$ & $1177(39.2 \%)$ & \\
\hline & Other & $10(18.2 \%)$ & $15(27.3 \%)$ & $6(10.9 \%)$ & $24(43.6 \%)$ & $55(1.8 \%)$ & \\
\hline & Not Recorded ${ }^{c}$ & $31(15.3 \%)$ & $42(20.7 \%)$ & $35(17.2 \%)$ & $95(46.8 \%)$ & $203(6.8 \%)$ & \\
\hline \multirow[t]{4}{*}{ Patient location $^{\mathrm{d}}$} & Within 25 miles & $265(18.0 \%)$ & $461(31.3 \%)$ & $201(13.6 \%)$ & $547(37.1 \%)$ & $1474(49.2 \%)$ & \multirow[t]{4}{*}{$<0.001^{\mathrm{b}}$} \\
\hline & Within 50 miles & $63(11.9 \%)$ & $168(31.7 \%)$ & $73(13.8 \%)$ & $226(42.6 \%)$ & $530(17.7 \%)$ & \\
\hline & $\begin{array}{l}\text { Other North } \\
\text { Carolina }\end{array}$ & $96(14.3 \%)$ & $236(35.1 \%)$ & $137(20.4 \%)$ & $203(30.2 \%)$ & $672(22.4 \%)$ & \\
\hline & Out of State & $30(9.3 \%)$ & $93(28.9 \%)$ & $62(19.3 \%)$ & $137(42.5 \%)$ & $322(10.7 \%)$ & \\
\hline \multirow[t]{4}{*}{ Marital status } & $\begin{array}{l}\text { Married/life } \\
\text { partner }\end{array}$ & $217(12.3 \%)$ & $602(34.2 \%)$ & $301(17.1 \%)$ & $641(36.4 \%)$ & $1761(58.7 \%)$ & \multirow[t]{4}{*}{$<0.001^{\mathrm{b}}$} \\
\hline & $\begin{array}{l}\text { Previously mar- } \\
\text { ried }\end{array}$ & $115(21.2 \%)$ & $151(27.9 \%)$ & $72(13.3 \%)$ & $204(37.6 \%)$ & $542(18.1 \%)$ & \\
\hline & Single & $108(18.5 \%)$ & $180(30.8 \%)$ & $87(14.9 \%)$ & $210(35.9 \%)$ & $585(19.5 \%)$ & \\
\hline & Not Recorded & $14(12.6 \%)$ & $25(22.5 \%)$ & $13(11.7 \%)$ & $59(53.2 \%)$ & $111(3.7 \%)$ & \\
\hline \multirow[t]{2}{*}{ Provider type } & $\mathrm{MD}$ & $227(11.2 \%)$ & $703(34.7 \%)$ & $373(18.4 \%)$ & $721(35.6 \%)$ & $2024(67.5 \%)$ & \multirow[t]{2}{*}{$<0.001^{\mathrm{b}}$} \\
\hline & APP & $227(23.3 \%)$ & $255(26.2 \%)$ & $100(10.3 \%)$ & $393(40.3 \%)$ & $975(32.5 \%)$ & \\
\hline
\end{tabular}

${ }^{\mathrm{a}}$ Wilcoxon

${ }^{\mathrm{b}}$ Chi-Square

${ }^{\mathrm{c}}$ Self-pay, uninsured, or insurance data were not available at the time of data extraction

${ }^{\mathrm{d}}$ Patient location missing for 1 visit attempt

Table 2 shows results of a multinomial logistic regression model for visits during the COVID period to identify factors associated with a completed telephone visit, completed video visit, or incomplete visit (canceled, no-show, or rescheduled). Age, race/ethnicity, insurance, marital status, and patient geographic location were all significant predictors of visit outcome. Specifically, Black race/ethnicity was associated with increased odds of completion of a telephone over a video visit, compared to White $(\mathrm{OR}=1.99,95 \%$ CI $1.47,2.68)$. When compared to private insurance, Medicaid, Medicare, Other, and Missing insurance status were all associated with increased odds of completing a telephone over a video visit, and Medicaid and Missing insurance type were also associated with increased odds of incomplete visits over a video visit. Compared to those currently married, being single or previously married (separated, divorced, widowed) were associated with increased odds of completing a telephone over a video visit.

To determine if these disparities applied to all completed visits, we evaluated the multinomial logistic regression model for any completed visit (telehealth or in-person) versus any incomplete visit (rescheduled, canceled, no-show) during the COVID period. We found that age, insurance status, and patient location were significantly associated with completed visits (Supplemental Table 2).

Patient satisfaction data from two patient experience surveys during the COVID period were analyzed by visit type. Among the patients who completed a telehealth visit $(N=1412$, phone, or video), only $12.5 \%$ responded to a survey. There was a statistically significant difference in patients' reported willingness to recommend the practice they visited, with patients completing video visits more 
Table 2 Multinomial logistic regression model for completed telephone visit or incomplete visit compared to completed video visit

\begin{tabular}{|c|c|c|c|c|c|}
\hline \multirow{2}{*}{$\begin{array}{l}\text { Category } \\
\text { Age }\end{array}$} & \multirow{2}{*}{$\begin{array}{l}\text { Variable } \\
\text { Per 1-year increase }\end{array}$} & \multirow{2}{*}{$\begin{array}{l}\text { Visit outcome (compared } \\
\text { to completed video visit) } \\
\text { Telephone }\end{array}$} & \multirow{2}{*}{$\begin{array}{l}\text { Odds ratio } \\
\mathbf{1 . 0 3}\end{array}$} & \multicolumn{2}{|c|}{$\begin{array}{l}95 \% \\
\text { confidence } \\
\text { limits }\end{array}$} \\
\hline & & & & 1.02 & 1.05 \\
\hline & & Incomplete & 1.03 & 1.02 & 1.03 \\
\hline \multirow[t]{2}{*}{ Race (vs. white) } & \multirow[t]{2}{*}{ Black } & Telephone & 1.99 & 1.47 & 2.68 \\
\hline & & Incomplete & 1.20 & 0.94 & 1.55 \\
\hline \multirow[t]{8}{*}{ Insurance (vs. private) } & \multirow[t]{2}{*}{ Medicaid } & Telephone & 4.51 & 2.81 & 7.26 \\
\hline & & Incomplete & 1.58 & 1.05 & 2.38 \\
\hline & \multirow[t]{2}{*}{ Medicare } & Telephone & 2.26 & 1.65 & 3.11 \\
\hline & & Incomplete & 1.15 & 0.92 & 1.44 \\
\hline & \multirow[t]{2}{*}{ Not recorded ${ }^{\mathrm{a}}$} & Telephone & 3.44 & 2.02 & 5.83 \\
\hline & & Incomplete & 2.45 & 1.65 & 3.65 \\
\hline & \multirow[t]{2}{*}{ Other insurance } & Telephone & 3.44 & 1.44 & 8.20 \\
\hline & & Incomplete & 1.78 & 0.89 & 3.52 \\
\hline \multirow{6}{*}{$\begin{array}{l}\text { Marital status (vs. mar- } \\
\text { ried or life partner) }\end{array}$} & \multirow[t]{2}{*}{ Previously married } & Telephone & 1.42 & 1.05 & 1.93 \\
\hline & & Incomplete & 1.12 & 0.87 & 1.44 \\
\hline & \multirow[t]{2}{*}{ Single } & Telephone & 1.69 & 1.21 & 2.35 \\
\hline & & Incomplete & 1.27 & 0.98 & 1.64 \\
\hline & \multirow[t]{2}{*}{ Not recorded } & Telephone & 1.35 & 0.66 & 2.76 \\
\hline & & Incomplete & 1.87 & 1.12 & 3.10 \\
\hline \multirow{4}{*}{$\begin{array}{l}\text { Patient location (vs. } \\
\text { within } 25 \text { miles of our } \\
\text { center) }\end{array}$} & \multirow[t]{2}{*}{ Other North Carolina } & Telephone & 0.79 & 0.59 & 1.07 \\
\hline & & Incomplete & 0.73 & 0.58 & 0.92 \\
\hline & \multirow[t]{2}{*}{ Within 50 miles } & Telephone & 0.65 & 0.46 & 0.91 \\
\hline & & Incomplete & 1.08 & 0.85 & 1.38 \\
\hline
\end{tabular}

Bold values indicate statistically significant Odds ratios

Not significant: sex, Hispanic versus non-Hispanic White race, non-Hispanic Other race versus non-Hispanic White race, unknown/not reported race versus Non-Hispanic White, out of state vs PSA

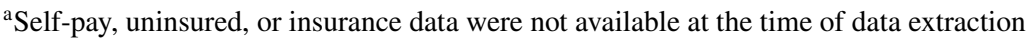

likely to recommend the practice compared to those completing telephone visits. There were no statistically significant differences in overall satisfaction or satisfaction with the provider based on type of visit (Supplemental Tables 3 and 4).

\section{Discussion}

The COVID-19 pandemic has placed stress on the ability of healthcare systems to provide adequate care for patients with liver disease. As telehealth practices have expanded throughout the country and globe to address this need, it is essential for liver providers to understand and identify gaps in this care approach to mitigate the risk and consequences of suboptimal care. Emerging evidence is showing that racial and ethnic minorities are disproportionately impacted by COVID-19, which is directly influenced by social determinants and barriers to care access [12]. The recent literature highlights a concern for inequity in telehealth access during the COVID-19 pandemic for chronic disease management in general [13], although little is known about care metrics in relation to liver disease management.

In this study, we sought to characterize liver-related care during the COVID-19 pandemic and found disparities in age, race/ethnicity, patient location, insurance, and marital status in the utilization of telehealth services at our center. In particular, telehealth visit attempts (video or telephone) involving Hispanic and Other patients were less likely to be completed, and video visit attempts of Black and Medicaid patients were less likely to be completed. Our findings potentially have implications for quality of care delivered. Although data on effectiveness of these visit types in hepatology are currently lacking, video visits may promote greater patient-physician communication and rapport, and also permit certain aspects of the physical exam to be performed including assessment of jaundice, sarcopenia, ascites, and hepatic encephalopathy. These factors may improve liver-related care to patients participating in video visits. Consistent with this hypothesis, patients at our center reported a greater willingness to recommend the practice if they completed a video visit, although other measures of satisfaction assessed in our patient satisfaction surveys 
did not differ by visit type. Given the above, facilitating video visits through improved or alternative means such as streamlined smartphone technology with reduced data burden could improve access, as could remote monitoring technologies such as those utilized for detection of hepatic encephalopathy [14].

Another reason identifying and addressing disparities in use of telehealth is crucial at this juncture is that telehealth may become a permanent fixture of disease management for patients with chronic liver disease, even after development of a COVID-19 vaccine. This will particularly be the case if payors continue to reimburse at rates equal or comparable to in-person visits. As such, ensuring that all patients may benefit equitably is important. Telehealth may actually mitigate some health care disparities by promoting equal access to subspecialty care for those with transportation or social support barriers. There are examples of positive effects on racial disparities from other diseases: for example, one remote monitoring program for diabetes narrowed the gap between hemoglobin A1c in Caucasians and African-Americans [15]. Telehealth may be beneficial to overall quality of care as well. Studies from the Veterans Health Administration have indicated that telehealth reduces the time from referral to evaluation for liver transplantation [16] and that telehealth triaging reduces the number of patients deemed not to be candidates during formal evaluation [17].

Though our study is the first to evaluate for usage disparities in liver telehealth during COVID-19, there are limitations. The retrospective design and limited dataset did not permit assessment of specific barriers to use of telehealth technologies on a patient-level (i.e., we did not have data on internet access, smartphone access, comfort with technology, or education level). In addition, data on reasons for rescheduling were not available. We did not have visit diagnoses (ICD-10 codes) for 95\% of visits occurring during the COVID period. Therefore, we were unable to evaluate whether differences in underlying conditions could have impacted patient access to telehealth or served as confounders. In addition, our categorization of race and insurance status means that there were groups with significant heterogeneity (for example, Not recorded insurance status, which included self-pay, uninsured, and patients for whom insurance information was not available). Patient satisfaction data were limited by low response rates to the surveys.

In summary, we found disparities in race, insurance, and marital status in utilization of telehealth visits for liver clinic in the early months of COVID-19. The morbidity and mortality of COVID-19 have disproportionately impacted minorities, who based on our data appear to have higher barriers to optimal telehealth care. Identifying populations and communities lacking infrastructure for telehealth will be an important aspect of preparing for future pandemics as well as long-term implementation of telehealth technology.
Health systems and communities will need to work together and be creative in circumnavigating the structural barriers to telehealth care in communities that lack these resources. Our study highlights potential populations where future work should focus. In addition, further studies investigating the effectiveness of different telehealth technologies, including patient outcomes such as hospitalization for exacerbation of chronic disease, should be undertaken to better understand whether certain technologies actually deliver better patient care.

Acknowledgments The authors gratefully acknowledge Phillip Maxwell and Desmond Cutler for their assistance with procuring visit data.

Author's contribution KW contributed to investigation, writing-original draft preparation. JMW, ZFG, and AJM were involved in conceptualization, writing-review and editing. AP and DN contributed to formal analysis, writing-review and editing. YAP was involved in conceptualization, investigation, supervision, writing-review and editing.

Funding Kara Wegermann is supported by NIH Grant 5T32DK00756827. Julius M. Wilder is supported by the 2018 AASLD Foundation grant Clinical, Translational, and Outcomes Research Award in Liver Transplantation.

\section{Compliance with Ethical Standards}

Conflict of interest The authors report no conflict of interest.

\section{References}

1. Verma S. Early impact Of CMS expansion of medicare telehealth during COVID-19 health affairs blog2020. Available from: https ://www.healthaffairs.org/do/10.1377/hblog20200715.454789/full. Accessed 24 Sep 2020.

2. Technology TOotNCfHI. Telemedicine and Telehealth 2017 [cited 2020]. Available from: https://www.healthit.gov/topic/health-itinitiatives/telemedicine-and-telehealth. Accessed 24 Sep 2020.

3. Tapper EB, Asrani SK. The COVID-19 pandemic will have a long-lasting impact on the quality of cirrhosis care. J Hepatol 2020;73:441-445. https://doi.org/10.1016/j.jhep.2020.04.005.

4. Serper M, Volk ML. Current and future applications of telemedicine to optimize the delivery of care in chronic liver disease. Clin Gastroenterol Hepatol 2018;16:157-161. https://doi. org/10.1016/j.cgh.2017.10.004.

5. Fix OK, Serper M. Telemedicine and telehepatology during the COVID-19 pandemic. Clin Liver Dis (Hoboken) 2020;15:187190. https://doi.org/10.1002/cld.971.

6. de Jong MJ, Boonen A, van der Meulen-de Jong AE, RombergCamps MJ, van Bodegraven AA, Mahmmod N et al. Cost-effectiveness of telemedicine-directed specialized vs standard care for patients with inflammatory bowel diseases in a randomized trial. Clin Gastroenterol Hepatol 2020;18:1744-52. https://doi. org/10.1016/j.cgh.2020.04.038.

7. Serper M, Shaked A, Olthoff KM, Hoteit M, Appolo B, Reddy KR. A local response to COVID-19 for advanced liver disease: current model of care, challenges and opportunities. J Hepatol. 2020. https://doi.org/10.1016/j.jhep.2020.05.022. 
8. Handschu R, Scibor M, Willaczek B, Nuckel M, Heckmann JG, Asshoff $\mathrm{D}$ et al. Telemedicine in acute stroke: remote videoexamination compared to simple telephone consultation. J Neurol 2008;255:1792-7. https://doi.org/10.1007/s00415-008-0066-9.

9. Rush KL, Howlett L, Munro A, Burton L. Videoconference compared to telephone in healthcare delivery: a systematic review. Int J Med Inform 2018;118:44-53. https://doi.org/10.1016/j.ijmed inf.2018.07.007.

10. Serper M, Nunes F, Ahmad N, Roberts D, Metz DC, Mehta SJ. Positive early patient and clinician experience with telemedicine in an academic gastroenterology practice during the COVID-19 pandemic. Gastroenterology 2020;159:1589-1591. https://doi. org/10.1053/j.gastro.2020.06.034.

11. Center PR. Internet/Broadband Fact Sheet 2019 [cited 2020]. Available from: https://www.pewresearch.org/internet/fact-sheet /internet-broadband/. Accessed 24 Sep 2020.

12. Wilder JM. The disproportionate impact of COVID-19 on racial and ethnic minorities in the United States. Clin Infect Dis. 2020. https://doi.org/10.1093/cid/ciaa959.

13. Sarah Nouri M, Khoong EC, Lyles CR, Karliner L. Addressing equity in telemedicine for chronic disease management during the Covid-19 pandemic. NEJM Catalyst. 2020. https://doi. org/10.1056/CAT.20.0123.

14. Bajaj JS, Thacker LR, Heuman DM, Fuchs M, Sterling RK, Sanyal AJ et al. The Stroop smartphone application is a short and valid method to screen for minimal hepatic encephalopathy. Hepatology 2013;58:1122-32. https://doi.org/10.1002/hep.26309.

15. Andersen JA, Scoggins D, Michaud T, Wan N, Wen M, Su D. Racial disparities in diabetes management outcomes: evidence from a remote patient monitoring program for type 2 diabetic patients. Telemed J E Health. 2020. https://doi.org/10.1089/ tmj.2019.0280.

16. John BV, Love E, Dahman B, Kurbanova N, Konjeti VR, Sundaram LT et al. Use of telehealth expedites evaluation and listing of patients referred for liver transplantation. Clin Gastroenterol Hepatol 2020;18:1822-1830. https://doi.org/10.1016/j. cgh.2019.12.021.

17. Konjeti VR, Heuman D, Bajaj JS, Gilles H, Fuchs M, Tarkington $\mathrm{P}$ et al. Telehealth-based evaluation identifies patients who are not candidates for liver transplantation. Clin Gastroenterol Hepatol 2019;17:207-209. https://doi.org/10.1016/j.cgh.2018.04.048.

Publisher's Note Springer Nature remains neutral with regard to jurisdictional claims in published maps and institutional affiliations. 